\title{
REPRODUCING KERNEL HILBERT SPACES FOR SPIKE TRAIN ANALYSIS
}

\author{
António R. C. Paiva ${ }^{1}$, Il Park ${ }^{2}$, and José C. Príncipe ${ }^{1 *}$ \\ ${ }^{1}$ Dept. of Electrical and Computer Engineering and ${ }^{2}$ Dept. of Biomedical Engineering \\ University of Florida \\ Gainesville, FL 32611, USA \\ \{arpaiva, memming, principe\}@cnel.ufl.edu
}

\begin{abstract}
This paper introduces a generalized cross-correlation (GCC) measure for spike train analysis derived from reproducing kernel Hilbert spaces (RKHS) theory. An estimator for GCC is derived that does not depend on binning or a specific kernel and it operates directly and efficiently on spike times. For instantaneous analysis as required for real-time use, an instantaneous estimator is proposed and proved to yield the GCC on average. We finalize with two experiments illustrating the usefulness of the techniques derived.
\end{abstract}

Index Terms - Spike train analysis, reproducing kernel Hilbert spaces, cross-correlation, synchrony detection.

\section{INTRODUCTION}

Correlation-based measures are widely used tools for spike train analysis [1]. In particular, the cross-correlation (CC) [2] is used to assess associations between neurons, or its normalized version to ease the statistical analysis [3]. However, because spike trains are point processes, in practice the estimation of the cross-correlation is not straightforward. A common approach is to bin the spike trains by moving a sliding window over time and counting the number of spikes in the window. In essence, binning is a transformation of randomness in time to randomness in amplitude, which is very attractive since it allows many of random process tools to be applied directly to the binned data. The main disadvantage is that binning inherently imposes time quantization on the spike times. This means that information will be lost if this information is contained in the precise spike times and/or their relative timing. Another major limitation is the fact that estimation of the cross-correlation assumes stationarity, which seems unlikely given the time-varying nature of neuronal function. Non-stationarity is often counteracted by performing the analysis in moving windows, but these windows must be kept small which greatly reduces the estimation accuracy. Finally, the analysis is only valid for pairs of neurons.

This paper discusses these issues and proposes an approach based on reproducing kernel Hilbert spaces (RKHS) to tackle them. The key observation is that the cross-correlation is a symmetric and positive definite kernel [4]. Thus, by the Moore-Aronsajn theorem [5], the CC kernel defines an RKHS where spike trains can be analyzed and processed systematically. However, we are also interested in addressing the limitations of the current methodology in the computation of the cross-correlation, therefore we analyze first its con-

* A. R. C. Paiva was supported by Fundação para a Ciência e a Tecnologia (FCT), Portugal, under grant SRFH/BD/18217/2004. This work was partially supported by NSF grant ECS-0422718 and CISE-0541241. struction, to reduce the cross-correlation to its essential ideas. Subsequently, we present the generalized cross-correlation (GCC) as an extended form and show how it gives rise to more effective ways to estimate the cross-correlation among two neurons. One of these tools is the instantaneous cross-correlation (ICC) which allows for single-trial estimation due to ensemble averaging.

\section{GENERALIZED CROSS-CORRELATION}

Binned spike trains are discrete-time signals. Therefore, the crosscorrelation is defined in the usual way as the expectation of the lagged product of the number of spikes per bin. Hence, assuming stationarity and ergodicity, the cross-correlation of binned spike trains $A$ and $B$ can be estimated with

$$
C_{A B}^{b i n}[l]=\frac{1}{M} \sum_{n=1}^{M} N_{A}[n] N_{B}[n+l],
$$

where $M$ is the number of bins and $N_{A}[n], N_{B}[n]$ are the number of spikes in the $n$th bin for spike train $A$ and $B$, respectively. Equation (1) clearly shows that $C_{A B}^{b i n}$ is an inner product of the binned spike trains. In RKHS theory the mapping into the RKHS is often unknown, but in this context it is readily noticeable that binning implements the mapping. However, binning of spike trains imposes quantization on the spike times and is therefore undesirable, especially when one is interested in analysis of the temporal structure in the form of synchrony as is the case here. This raises the question of what is binning actually doing? And, correspondingly, can we utilize a better way to do it? Both these issues are addressed in this section.

As shown by Dayan and Abbott [6], binning is an estimator of the instantaneous firing rate (apart from a normalization by the bin size). Hence, a more general form of inner product, which we shall call generalized cross-correlation (GCC), can be defined directly in terms of the intensity functions of the point processes,

$$
\begin{aligned}
C_{A B}(\theta) & =E\left\{\lambda_{A}(t) \lambda_{B}(t+\theta)\right\} \\
& =\lim _{T \rightarrow \infty} \frac{1}{2 T} \int_{-T}^{T} \lambda_{A}(t) \lambda_{B}(t+\theta) d t,
\end{aligned}
$$

where $\lambda_{A}(t)$ and $\lambda_{B}(t)$ denote the intensity functions of spike trains $A$ and $B$, respectively. This is a functional inner product in an infinite dimensional space. We might think that $C_{A B}^{b i n}$ is finite dimensional approximation of this functional measure.

In the statistical literature the conventional approach for intensity function estimation of point processes is kernel smoothing [7], with clear advantages in the estimation [8]. Denote the spike times 
of a spike train, say, $A$, in the time interval $[0, T]$ as $\left\{t_{i}^{A}: i=\right.$ $\left.1, \ldots, N_{A}\right\}$, where $N_{A}$ is the number of spikes of $A$ in the interval. The kernel estimated intensity function is given by

$$
\hat{\lambda}_{A}(t)=\sum_{m=1}^{N_{A}} h\left(t-t_{m}^{A}\right)
$$

where $h$ is the smoothing kernel function. For intensity estimation this function $h$ must integrate to one. Substituting these intensity estimations in the definition of the inner product in Eq. (2) and restricting the evaluation to the $[0, T]$ interval yields an estimator for the GCC

$$
\hat{C}_{A B}(\theta)=\frac{1}{T} \sum_{m=1}^{N_{A}} \sum_{n=1}^{N_{B}} \kappa_{\tau}\left(t_{m}^{A}-t_{n}^{B}+\theta\right),
$$

where $\kappa_{\tau}$ is the kernel obtained by the convolution of the intensity estimation kernel $h$ with itself, and $\tau$ is the kernel size (or bandwidth) parameter. Notice that $C_{A B}^{b i n}$ is a special case of Eq. (4) in which the spike times are first quantized and then the GCC evaluated with a rectangular kernel.

Unlike the cross-correlation of binned spike trains, the GCC estimator operates directly on the spike times with full resolution. It also takes advantage of the typically sparse nature of spike trains for efficient evaluation. Consequently, the computational complexity depends on the firing rate of the neurons and not on the sampling rate. This estimator allows, for example, to construct continuoustime cross-correlograms with much higher precision and in a fraction of the time required by explicitly smoothing [9].

A number of kernels $\kappa$ may be used in the GCC estimator. However, $\kappa$ must integrate to one (since so does $h$ ) and be symmetric and positive definite. Then, it is easy to prove that the GCC estimator is also a symmetric and positive definite kernel function, and therefore denotes a well defined inner product. Thus, $\hat{C}_{A B}$ induces an RKHS for the manipulation and analysis of spike trains. Notice that Eq. (3) can be interpreted as the convolution of the spike train with a window given by the smoothing function $h$, therefore the parameter $\tau$ determines the smoothing introduced by $h$ and the kernel, and thus regulates the scale at which the GCC estimator interprets an intensity function, between the extremes of synchrony in neuron firings (for small kernel size) or firing rate (large kernel size).

\section{INSTANTANEOUS CROSS-CORRELATION}

The GCC is a more general form of cross-correlation that does not require binning but it still needs a window of data to operate. It is therefore still dependent on piecewise stationary assumption. As a function of time, the integrand of the GCC (Eq. (2)), called the instantaneous cross-correlation (ICC), provides a more appropriate representation. ICC is a continuous function of the spike timings and describes temporal structure of the inhomogeneous firings allowing for a direct assessment of similarity in time. One might think of it as a scalar inner product along each of the dimensions indexed by time.

Since we seek methodologies that can be applied online, only causal intensity estimation kernels can be considered. We propose to use the exponential function,

$$
h(t)=(1 / \tau) \exp [-t / \tau] u(t),
$$

where $u(\cdot)$ is the step function. The exponential function provides both graded interactions and a time scale for the intensity estimation by controlling the time constant $\tau$. The ideas presented are not limited to Eq. (5), but it was chosen for its biological plausibility since it can be interpreted as evoked post-synaptic potentials in a neuron, its wide use throughout neuroscience [6], and its computational simplicity.

Using the exponential function, at time $t$ the estimated intensity function of spike train $A$ is given as

$$
\hat{\lambda}_{A}(t)=\frac{1}{\tau} \sum_{t_{m}^{A} \leq t} \exp \left(-\frac{t-t_{m}^{A}}{\tau}\right) u\left(t-t_{m}^{A}\right) .
$$

This is nothing but the filtering of a spike train by a first order IIR filter. Then, the ICC is given simply by

$$
\tilde{c}_{A B}(t, \theta)=\hat{\lambda}_{A}(t) \hat{\lambda}_{B}(t+\theta) .
$$

Notice that this two layer evaluation process can be computed very easily, and is especially suited for hardware implementation.

For small values of $\tau$ the ICC quantifies statistically our intuition of synchrony, graded with the decaying exponential function and followed by a coincidence detection operator implemented by the product. When two neurons spike synchronously the product of the estimated intensities at that time will be high, with a maximum if they spike exactly at the same time. But, if the spikes are separated by more than $\approx 5 \tau$ then the ICC is nearly zero. In this respect, the ICC resembles the "gravity force" in the gravity transform framework $[10,11]$, but the present work provides a statistical interpretation for the estimator and much broader perspective not available before.

As the formulation of ICC suggests, $\tilde{c}_{A B}$ is a stochastic approximation of the GCC under stationarity. This is obvious by taking the expectation of Eq. (7) over time. In particular, the average ICC over a time interval $[0, T]$ with a exponential function results in

$$
\begin{aligned}
\frac{1}{T} \int_{0}^{\infty} \tilde{c}_{A B}(t, \theta) d t & =\frac{1}{T} \sum_{m=1}^{N_{A}} \sum_{n=1}^{N_{B}} \frac{1}{2 \tau} \exp \left(-\frac{\left|t_{m}^{A}-t_{n}^{B}+\theta\right|}{\tau}\right) \\
& =\frac{1}{T} \sum_{m=1}^{N_{A}} \sum_{n=1}^{N_{B}} \kappa_{\tau}\left(t_{m}^{A}-t_{n}^{B}+\theta\right) \\
& =\hat{C}_{A B}(\theta)
\end{aligned}
$$

where, in this case, $\kappa_{\tau}$ denotes the Laplacian kernel. (The integration goes up to infinity to account for the infinite support of the exponential function but only spike times before $T$ are included.) Note that the exponential function gives rise to the Laplacian kernel which verifies all the requirements for $\hat{C}_{A B}$ to represent a well defined inner product.

\subsection{ICC as a neural ensemble measure}

The ICC exploits the temporal nature of the spike trains and enables instantaneous estimation of synchrony because no temporal averaging is done. The price paid is that, for a single pairs of neurons, variability in the spike times is directly translated into the ICC and thus its estimation is quite "noisy" due to events occurring by chance. Instead of averaging ICC over time which yields the GCC in a time interval, an alternative way to reduce the variance of this estimator is to compute the expectation over the neural ensemble,

$$
\bar{c}(t, \theta)=E\left\{\tilde{c}_{A B}(t, \theta)\right\},
$$




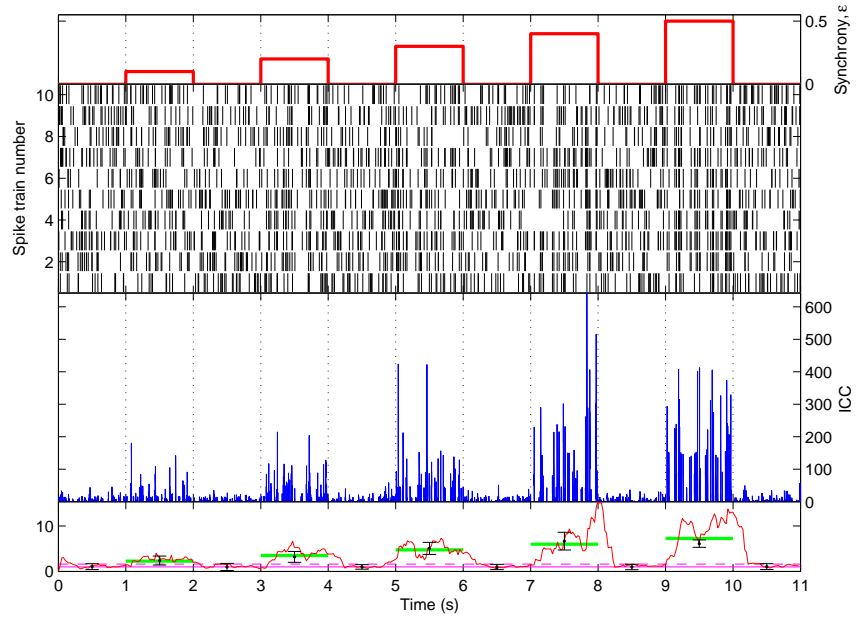

Fig. 1. Analysis of ICC as a function of synchrony. (Top) Level of synchrony used in the simulation of spike trains. (Upper middle) Raster plot of firings. (Lower middle) Ensemble averaged ICC. (Bottom) Time average of ICC in the upper plot computed with a causal rectangular window $250 \mathrm{~ms}$ long in steps of $25 \mathrm{~ms}$ (dark gray). For reference, it is also displayed the expected value (dashed line) and this value plus two standard deviations (dotted line) for independent neurons, together with the expected value during moments of synchronous activity (thick light gray line), as obtained analytically from the level of synchrony used in the generation of the dataset. Furthermore, the mean and standard deviation of the ensemble averaged GCC scaled by $T$ measured from data in one second intervals is also shown (black).

where $E\{\cdot\}$ denotes the expectation over all pairs of neurons.

The ensemble averaged ICC is a spatio-temporal measure of the ensemble cooperation over time. In this form, and due to the exchange of time for ensemble averaging, the ICC is capable of detecting the presence of dynamic cell assemblies in the ensemble with high temporal resolution. However it raises the problem of neural selection to evaluate the ensemble average.

\section{EXPERIMENTS}

\subsection{ICC as a synchronization measure}

For this example, we generated 10 homogeneous spike trains using the multiple interaction process (MIP) [12]. The MIP model allows for multiple spike trains to be generated according to a selected synchrony level, $\varepsilon$, which is the count correlation coefficient and quantifies the probability of a spike co-occurrence in another spike train.

Figure 1 shows one realization of the generated spike trains with varying levels of synchrony. All simulated spike trains have average firing rate 20 spikes/s. The figure shows the ICC averaged for each time instant over all pair combinations of spike trains. The time constant, $\tau$, of the exponential for intensity estimation was chosen to be $2 \mathrm{~ms}$. To verify Eq. (8), the bottom plot shows the average value of the mean ICC. This was computed with a causal $250 \mathrm{~ms}$ long sliding window in $25 \mathrm{~ms}$ steps. To establish a relevance of the values shown, the expectation and the expectation plus two standard deviations are also shown, assuming independence between spike trains. The mean and standard deviation, assuming independence, are 1 and $\sqrt{\left(\frac{1}{2 \tau \lambda}+1\right)^{2}-1}$, respectively. The expected value of the

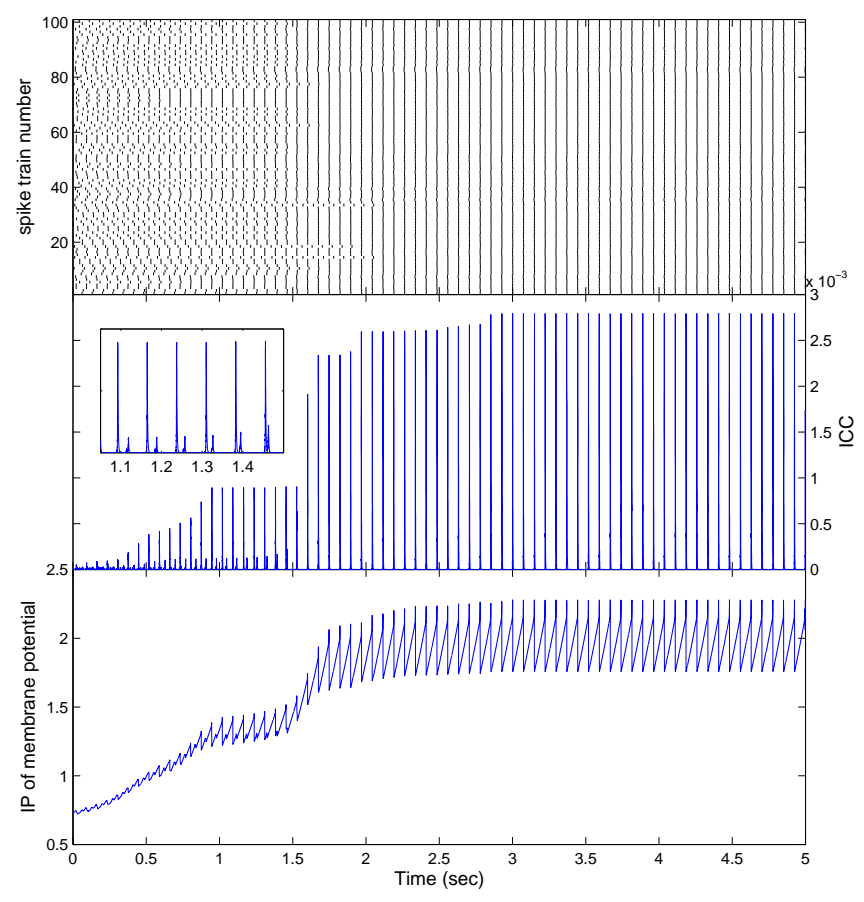

Fig. 2. Evolution of synchrony in a spiking neural network. (Top) Raster plot of the neuron firings. (Middle) ICC over time. The inset highlights the merging of two synchronous groups. (Bottom) Information potential of the membrane potentials. This is a macroscopic variable describing the synchrony in the neurons' internal state.

ICC for a given synchrony level is $1+\varepsilon /(2 \tau \lambda)$, with $\lambda$ the firing rate of the two spike trains, and is also shown in the plot for reference. Finally, the ensemble averaged GCC computed for each second of data is also shown.

It is noticeable from the figure that the ICC estimated synchrony increases as measured by ICC. Moreover, the averaged ICC is very close to the theoretical expected value and is typically below the statistical upper bound under an independence assumption as given by the line indicating the expectation plus two standard deviations. The delayed increase in the averaged ICC is a consequence of the causal averaging of ICC. It is equally remarkable to verify that GCC matches precisely the expected values from ICC as given analytically. This shows a significant advantage of the proposed GCC/ICC framework as it can be used for analysis of data providing not only detection ability but also the possibility to actually measure the synchrony level with a high degree of accuracy.

\subsection{Synchronization of pulse-coupled oscillators}

In this example, we show that ICC can quantify synchrony in a spiking neural network of leaky-integrate-and-fire (LIF) neurons designed according to Mirollo and Strogatz [13 $]^{1}$ and the ICC results compare favorably with the extended cross-correlation for multiple neurons. The network is initialized in a random condition and is proven to synchronize over time (Fig. 2). The synchronization is

\footnotetext{
${ }^{1}$ The parameters for the simulation are: 100 neurons, resting and reset membrane potential $-60 \mathrm{mV}$, threshold $-45 \mathrm{mV}$, membrane capacitance 300 $\mathrm{nF}$, membrane resistance $1 \mathrm{M} \Omega$, current injection $50 \mathrm{nA}$, synaptic weight 100 $\mathrm{nV}$, synaptic time constant $0.1 \mathrm{~ms}$ and all to all excitatory connection.
} 

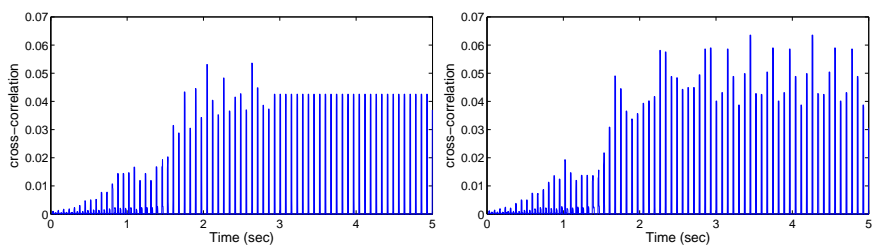

Fig. 3. Zero-lag cross-correlation computed over time using a sliding window 10 bins long, and bin size $1 \mathrm{~ms}$ (top) and $1.1 \mathrm{~ms}$ (bottom).

essentially due to leakiness and the weak coupling among the oscillatory neurons.

The raster plot of neuron firings is shown in Fig. 2. There are two main observations: the progressive synchronization of the firings associated with the global oscillatory behavior of the network, and the local grouping that tends to preserve local synchronizations that either entrain the full network or wash out over time, as expected from theoretical studies of the network behavior [13]. The ICC depicts this behavior precisely: the synchronization increases monotonically, with a period of fast increase in the first second followed by a plateau and slower increase as time advances. Moreover, it is possible to observe in the first $1.5 \mathrm{~s}$ the formation of a second group of synchronized neurons which slowly merges into the main group. In addition, the envelope of ICC reveals the coherence in the membrane potentials quantified by the information potential (IP). The IP is an information theoretic quantity inversely proportional to entropy [14]. It was computed with $\mathrm{IP}_{\theta}=\frac{1}{M^{2}} \sum_{i=1}^{M} \sum_{j=1}^{M} \exp \left(-d\left(\theta_{i}, \theta_{j}\right) / 2 \sigma^{2}\right)$ with $\sigma=75 \mathrm{mV}^{2}$ The IP measures synchrony of the neuron's internal state, which is only available in simulated networks. Yet the results show that ICC was able to successfully and accurately extract such information from the observed spike trains.

In Fig. 3 we also present the zero-lag cross-correlation over time, averaged through all pairwise combinations of neurons. The crosscorrelation was computed with a sliding window 10 bins long, sliding 1 bin at a time. Results are shown for bin sizes of $1 \mathrm{~ms}$ and $1.1 \mathrm{~ms}$. It is notable that although cross-correlation captures the general trends of synchrony, it masks the plateau and the final synchrony and it is highly sensitive to the bin size as shown in the figure, unlike ICC. In other words, the results for the windowed cross-correlation highlight the importance of working in "continuous" time and without time averaging for robust spike train analysis.

\section{DISCUSSION}

In this paper we utilized RKHS ideas to define cross-correlation between spike trains in the form of an inner product between their intensity functions. This result generalizes the often used crosscorrelation of binned spike trains. In addition, the formulation presented yields a simple estimator with better accuracy since binning is avoided.

From a more practical perspective, we derived an instantaneous estimator of the GCC, called the ICC, and proved its unbiasedness. ICC is practically useful in multichannel spike train recordings because it is a measure of the similarity in temporal structure across the ensemble of neurons. Because it is estimated in continuous time and therefore without discretization error normally incurred with bin-

\footnotetext{
${ }^{2}$ The distance used in the Gaussian kernel was $d\left(\theta_{i}, \theta_{j}\right)=$ $\min \left(\left|\theta_{i}-\theta_{j}\right|, 15 m V-\left|\theta_{i}-\theta_{j}\right|\right)$, where $\theta_{i}$ is the membrane potential of the $i$ th neuron. This wrap-around effect expresses the phase proximity of the neurons before and after firing.
}

ning, higher temporal resolution is achieved. Particularly in the examples shown, we chose to illustrate the use of ICC to characterize temporal structure in the form of synchronous firings. The disadvantage in the ensemble average approach however is that it requires knowledge a priori of the neurons that belong to an ensemble. We are currently working on this problem and hypothesize the solution might involve clustering of neurons or PCA of their responses.

This paper focuses on how the RKHS can be utilized to better analyze spike trains. It must be remarked however that the establishment of an RKHS associated with the cross-correlation is perhaps of even higher importance for the derivation of more principled algorithms to process spike trains.

\section{REFERENCES}

[1] Emery N. Brown, Robert E. Kass, and Partha P. Mitra, "Multiple neural spike train data analysis: state-of-the-art and future challenges," Nature Neurosci., vol. 7, pp. 456-461, 2004.

[2] D. H. Perkel, G. L. Gerstein, and G. P. Moore, "Neuronal spike trains and stochastic point processes. II. simultaneous spike trains.," Biophys. J., vol. 7, no. 4, pp. 419-440, July 1967.

[3] Carlos D. Brody, "Correlations without synchrony," Neural Comp., vol. 11, no. 7, pp. 1537-1551, 1999.

[4] Emanuel Parzen, "Statistical inference on time series by Hilbert space methods," Tech. Rep. 23, Applied Mathematics and Statistics Laboratory, Stanford University, Stanford, California, Jan. 1959.

[5] N. Aronszajn, "Theory of reproducing kernels," Trans. Am. Math. Soc., vol. 68, no. 3, pp. 337-404, May 1950.

[6] Peter Dayan and L. F. Abbott, Theoretical Neuroscience: Computational and Mathematical Modeling of Neural Systems, MIT Press, Cambridge, MA, USA, 2001.

[7] Rolf-Dieter Reiss, A Course on Point Processes, SpringerVerlag, New York, NY, 1993.

[8] Robert E. Kass, Valérie Ventura, and Can Cai, "Statistical smoothing of neuronal data," Network: Comp. Neural Sys., vol. 14, pp. 5-15, 2003.

[9] Il Park, António R. C. Paiva, Thomas B. DeMarse, and José C. Príncipe, "An efficient algorithm for continuous-time cross correlation of spike trains," J. Neurosci. methods, 2007, submitted.

[10] George L. Gerstein, Donald H. Perkel, and Judith E. Dayhoff, "Cooperative firing activity in simultaneously recorded populations of neurons: detection and measurement," J. Neurosci., vol. 5, no. 4, pp. 881-889, 1985.

[11] George L. Gerstein and Ad M. Aertsen, "Representation of cooperative firing activity among simultaneously recorded neurons," J. Neurophysiol., vol. 54, no. 6, pp. 1513-1528, 1985.

[12] Alexandre Kuhn, Ad Aertsen, and Stefan Rotter, "Higher-order statistics of input ensembles and the response of simple model neurons.," Neural Comp., vol. 15, no. 1, pp. 67-101, 2003.

[13] Renato E. Mirollo and Steven H. Strogatz, "Synchronization of pulse-coupled biological oscillators," SIAM J. Applied Math., vol. 50, no. 6, pp. 1645-1662, Dec. 1990.

[14] José C. Príncipe, Dongxin Xu, and John W. Fisher, "Information theoretic learning," in Unsupervised Adaptive Filtering, Simon Haykin, Ed., vol. 2, pp. 265-319. John Wiley \& Sons, 2000. 